\title{
Non-Hodgkin's lymphoma: A case of testis lymphoma metastasized to the gallbladder
}

\author{
Yutaka Tsutsumi $^{1 *}$, Reiki Ogasawara ${ }^{1}$, Fumiyasu Yamamoto $^{2}$, Yutaka Yamada ${ }^{3}$, Yasuyuki Komatsu ${ }^{1}$, \\ Kazuhiko Ichiki $^{1}$, Shinich Ito ${ }^{1}$, Nobuaki Kurauchi ${ }^{4}$, Kazuhiro Kudo ${ }^{5}$, Norihiko Shimoyama ${ }^{5}$, \\ Junji Tanaka $^{6}$, Masahiro Asaka ${ }^{7}$, Masahiro Imamura ${ }^{6}$, Hirohito Naruse ${ }^{2}$, Nobuo Masauzi ${ }^{1}$ \\ ${ }^{1}$ Department of Internal Medicine, Hakodate Municipal Hospital, Hakodate, Japan \\ ${ }^{2}$ Department of Gastroenterology, Hakodate Municipal Hospital, Hakodate, Japan \\ ${ }^{3}$ Department of Urology, Hakodate Municipal Hospital, Hakodate, Japan \\ ${ }^{4}$ Department of Surgery, Hakodate Municipal Hospital, Hakodate, Japan \\ ${ }^{5}$ Department of Clinical Pathology, Hakodate Municipal Hospital, Hakodate, Japan \\ ${ }^{6}$ Department of Hematology and Oncology, Graduate School of Medicine, Hokkaido University, Sapporo, Japan \\ ${ }^{7}$ Department of Gastroentelology, Graduate School of Medicine, Hokkaido University, Sapporo, Japan \\ Email: *yutsutsu@shore.ocn.ne.jp
}

Received 1 December 2011; revised 17 January 2012; accepted 29 January 2012

\begin{abstract}
Testis lymphoma (diffuse large B cell lymphoma) metastasized to the gallbladder. The gall bladder is rarely involved in cases of the malignant lymphoma, and lymphoma metastasis from the testis to the gallbladder has not been previously reported. These reports may possibly change by the introduction of PET/CT. Furthermore, although it was a struggle to diagnose this particular case, there is a possibility that we may have been able to recognize metastasis from the testis at an earlier stage if PET/CT had been conducted.
\end{abstract}

Keywords: Testis Lymphoma; Gallbladder; PET/CT

A 70-year-old male was hospitalized on 19 May 2008 for swelling of the left testis without pain. He had no history of gallstones, anorexia, weight loss, jaundice, fever, or abdominal tenderness. Laboratory findings showed a leukocyte count of $7.6 \times 10^{9} \mathrm{~L}$, a total bilirubin of 0.4 $\mathrm{mg} / \mathrm{dl}$, an AST of $20 \mathrm{IU} / \mathrm{L}$, an ALT of $35 \mathrm{IU} / \mathrm{L}$, an alkaline phosphatase of $315 \mathrm{IU} / \mathrm{L}$, and a serum interleukin-2 receptor level of $574 \mathrm{U} / \mathrm{mL}$. A left testis tumor and a gallbladder tumor were observed in a computed tomography (CT) scan (Figure 1). Left-side orchiectomy was performed on 26 May 2008, and cytological examination of the testis indicated lymphoma invasion (Figure 2). The gallbladder tumor was expected to be cancer of the gallbladder, therefore, ultrasonic endoscope, blood angiography, ERCP, and bile cytology were used for the diagnosis. Bile cy-tology predicted a malignant lymphoma, but did

\footnotetext{
"Corresponding author.
}

not confirm the tissue-type. In addition, these exams did not find gallstones. Positron emission tomography (PET) was performed after these examinations and the result strongly indicated the aggressive lymphoma of gall bladder (Figure 1(c)). Therefore, Cholecystectomy was performed on 23 June 2008, and the pathological finding was diffuse large B-cell lymphoma (Figure 3). Specimens from both the testis and gallbladder were positive for CD20, CD79a, bcl6, and MUM1, and the patient was diagnosed with diffuse large B-cell lymphoma of the testis and metastatic lymphoma of the gall bladder. He was treated with inverted-Y radiation therapy and six courses of R-THP-COP therapy $\left(375 \mathrm{mg} / \mathrm{m}^{2}\right.$ rituximab on day $1 ; 500 \mathrm{mg} / \mathrm{m}^{2}$ cyclophosphamide, $30 \mathrm{mg} / \mathrm{m}^{2}$ pirarubicin, and $1 \mathrm{mg} / \mathrm{m}^{2}$ vincristine on day2; and $30 \mathrm{mg} / \mathrm{m}^{2}$ prednisolone on days 2 - 6). The patient was in complete remission on 1 July 2009. Periodic surveillance examinations with PET/CT were performed on the $22^{\text {nd }}$ of March 2010 and multiple lymphoid lesions were observed. PET standard uptake value (SUV) showed diffuse large B cell lymphoma disease recurrence. An identical therapeutic regimen of R-THP-COP was performed (six courses) from the $31^{\text {st }}$ of March 2010. Patients experienced a second remission on the $17^{\text {th }}$ of September.

Non-Hodgkin's lymphoma (NHL) is a rare disease, accounting for only about $5 \%$ of all testicular neoplasms. Primary NHL of the testis has a poor prognosis, spreading to unusual extra-nodal sites and recurring after remission. This disease has a marked tendency to metastasize to the skin, lung, central nervous system, and contralateral testis [1]. Involvement of the gallbladder is rare in cases of malignant lymphoma, and metastasis of malignant lymphoma from the testis to the gallbladder has 


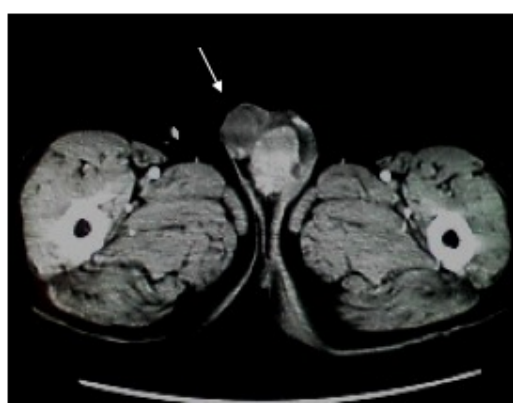

(a)

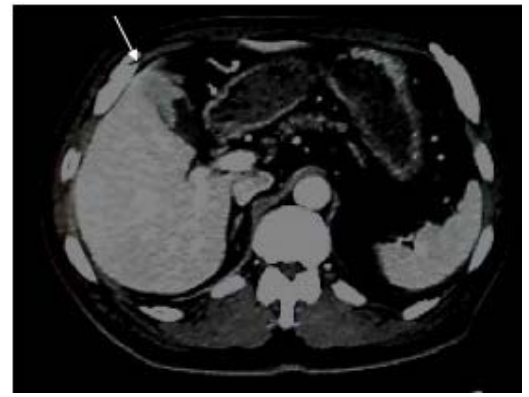

(b)

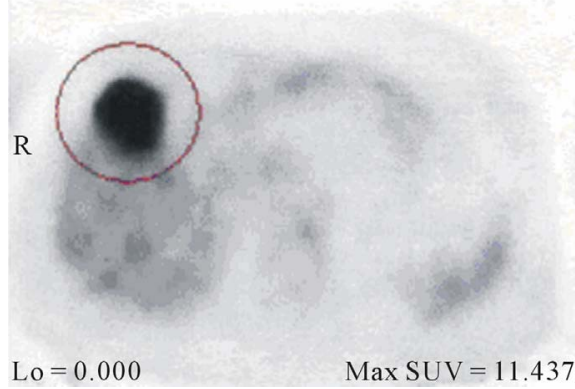

(c)

Figure 1. (a), (b) CT scans showing the left testis tumor and the dense soft tissue mass in the gallbladder; (c) PET showing the high SUV lesion in gall bladder.

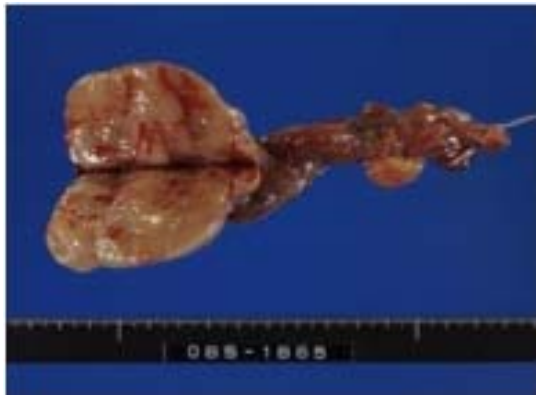

(a)

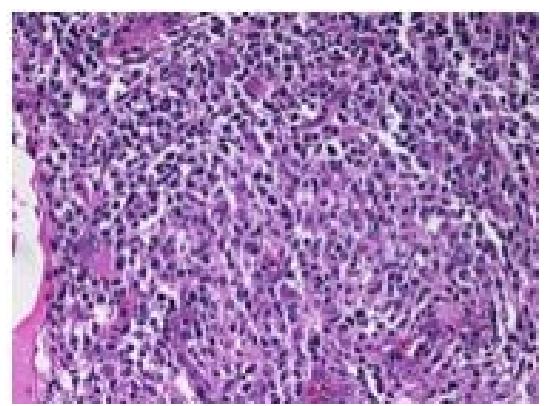

(b)

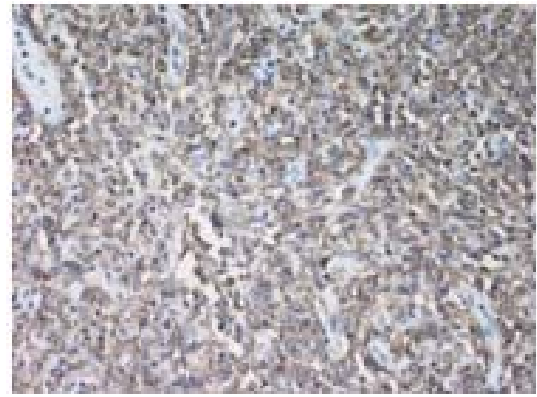

(c)

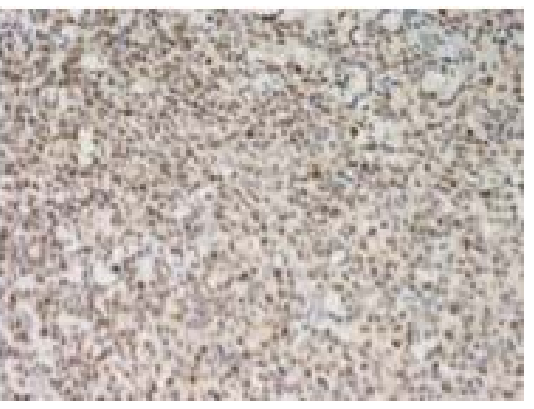

(d)

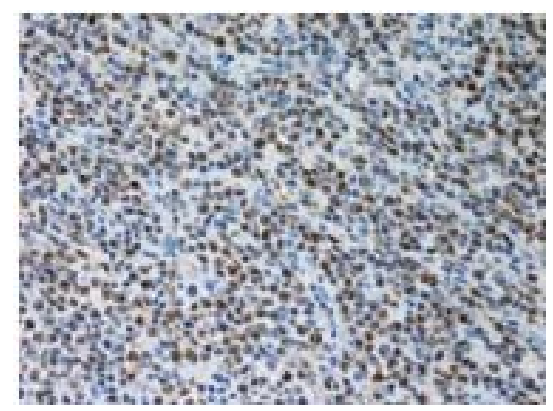

(e)

Figure 2. (a) Testis swelling due to the NHL; (b) Microscope image showing the testis diffusely infiltrated with malignant cells; (c)-(e) These cells were positive for CD20, bcl-6, and MUM1.

not been reported previously [2,3]. Lymphoma metastasis is a complex series of the steps in which lymphoma cells leave the primary tumor and migrate to other parts of the body via the blood stream of the lymphatic system. A testis lymphoma might spread to non-lymphoid organs without lymphoid lesions at early stage of the disease progression. The fact that the present testis lymphoma is able to spread to an extra-nodal site might show the bloodstream was part of metastatic system for this tumor. On the other hand, only lymphoid lesions were observed in disease-recurrent cases. Various characteristic changes were suspected, however, lymph-node analysis was not performed at the time of recurrence.

Recently, PET examination has been able to distinguish various types of malignant neoplasms [4]. PET/CT is now a standard procedural tool used for staging malignant lymphomas. A strong association between residual ${ }^{18}$ F-fluorodeoxy glucose (FDG) positivity and poor prognosis has also been established [5]. Furthermore, FDG-PET performed early during treatment may predict the prognosis of lymphoma patients [5-7]. PET is also able to detect the existence of multiple organ neoplasms, including kidney, pancreas, esophagus, hepatobiliary, uterine, ovary, and others [4]. Although detection of primary and metastatic gall bladder lymphomas is currently rare, the rate of detection may increase with concurrent use of PET/CT procedures. If PET/CT had been performed initially in this case, metastatic lymphoma of the gall bladder might have been diagnosed more easily. Gall bladder tumor may be suspected in instances of 


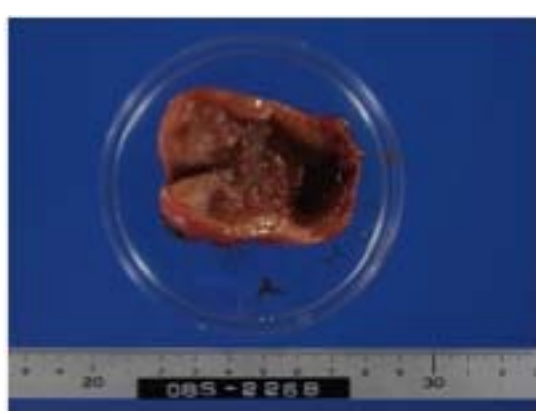

(a)

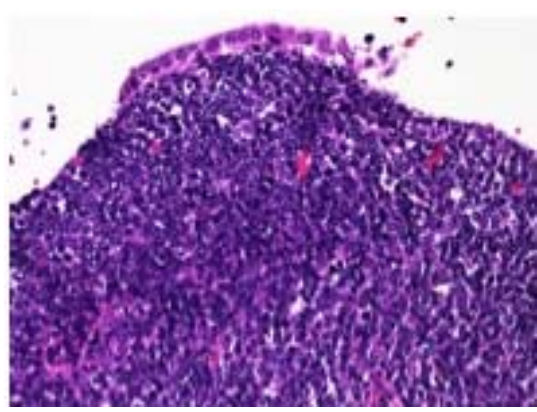

(b)

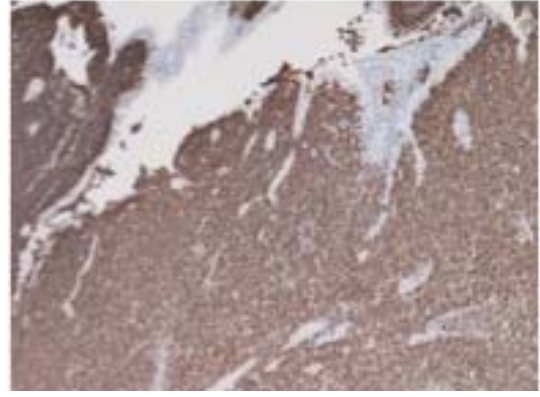

(c)

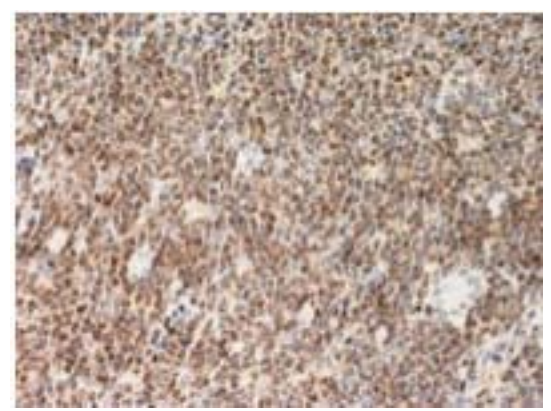

(d)

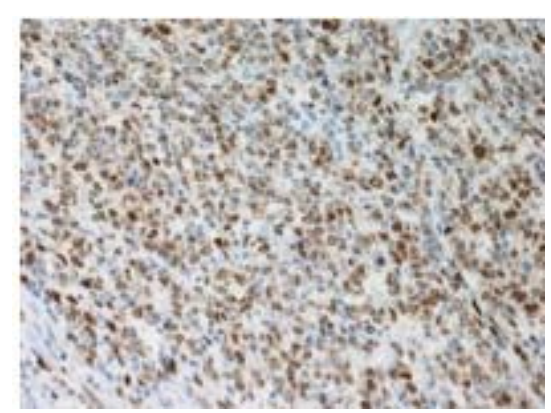

(e)

Figure 3. (a) Gallbladder showed wall thickness due to the NHL; cells were positive for CD20, bcl-6, and MUM1.

lymphoma originating in distant regions. PET/CT will be of great assistance in diagnosis and staging, particularly in these rare cases of disease progression. In addition, the technique allows the avoidance of highly invasive exams and surgeries and is expected to become a powerful tool in diagnosis and disease staging. Unfortunately, given that complications of diffuse large B cell lymphoma and gallbladder lymphomas have been reported, cholecystectomy may not be completely avoided [8]. Distinctive characteristics exist in different types of lymphomas. While gallstones are a common complication in cases of primary gallbladder lymphomas, aged individuals suffer from diffuse large B cell lymphoma and malt lymphoma relatively more often, which would be expected to help in differentiating metastatic lymphomas from these two types [9]. In addition, it has been reported that both PET and SUV can detect the differences between low-grade and aggressive lymphomas, therefore, it would help in the evaluation of either the complications of a primary gallbladder lymphoma or metastasis [10]. In the future, more detailed characteristics of gallbladder lymphomas (including primary and metastatic), and their relation to other lymphomas, are expected to become clearer.

\section{REFERENCES}

[1] Zucca, E., Conconi, A., Mughal, T.I., et al. (2003) Patterns of outcome and prognostic factors in primary large-cell lymphoma of the testis in a survey by the international extranodal lymphoma study group. Journal of Clinical Oncology, 21, 20-27. doi:10.1200/JCO.2003.11.141

[2] Mitropoulous, F.A., Angelopoulou, M.P., Siakantaris, G.R., et al. (2000) Primary non-Hodgkin's lymphoma of the gallbladder. Leukemia \& Lymphoma, 40, 123-131.

[3] Repine, T.B., DeArmond, G. and Lopez, J.D. (2004) Burkitt's lymphoma involving the gallbladder. Journal of Clinical Oncology, 22, 5014-5015. doi:10.1200/JCO.2004.11.026

[4] Minamimoto, R., Senda, M., Terauchi, T., et al. (2010) Analysis of various malignant neoplasm detected by FDG-PET cancer screening program: Based on a Japanese Nationwide Survey. Annals of Nuclear Medicine, 17, 45-54.

[5] Michaellet, A.S., Trotman, J. and Tychyl-Pinel, C. (2010) Role of early PET in the management of diffuse large B cell lymphoma. Current Opinion in Oncology, 22, 414418. doi:10.1097/CCO.0b013e32833d5905

[6] Lin, C., Itti, E., Haioun, C., et al. (2007) Early 18F-FDG PET for Prediction of Prognosis in Patients with diffuse large B cell lymphoma: SUV-based assessment versus visual analysis. Journal of Nuclear Medicine, 10, 16261632.

[7] Ng, A.P., Seymour, J.F., Lee, M., et al. (2007) Early therapeutic response assessment by (18) FDG-positron emission tomography during chemotherapy I patients with diffuse large B-cell lymphoma: Isolated residual positivity involving bone is not usually a predictor of subsequent treatment failure. Leukemia \& Lymphoma, 48, 
596-600. doi:10.1080/10428190601099965

[8] Gardini, A., Saragori, L., La Barba, G., et al. (2009) Simultaneous occurrence of primary diffuse large B cell lymphoma and extranodal marginal zone (MALT) B-cell lymphoma in the gallbladder: A case report. Pathologica, 101, 230-234.

[9] Mani, H., Climent, F., Colomo, L., et al. (2010) Gall bladder and extra hepatic bile duct lymphomas: Clinicopathological observations and biological implications. Am
Journal of Surgical Pathology, 34, 1277-1286. doi:10.1097/PAS.0b013e3181e9bb8b

[10] Schoder, H., Noy, A., Gonen, M., et al. (2005) Intensity of 18fluorodeoxyglucose uptake in positron emission tomography distinguishes between indolent and aggressive non-Hodgkin's lymphoma. Journal of Clinical Oncology, 23, 4643-4651. doi:10.1200/JCO.2005.12.072 